\title{
Análisis de la técnica y estrategia de paso en piragüismo de aguas tranquilas Technique analysis and pacing strategy in flatwater canoeing
}

\author{
Fernando Alacid Cárceles* \\ *Universidad de Murcia (España)
}

\begin{abstract}
Resumen: El objetivo de este trabajo fue describir las tendencias en análisis de la técnica de paleo y en las estrategias de paso en piragüismo de aguas tranquilas. El análisis cualitativo se describió a través de las valoraciones descriptiva y discriminativa, mientras que el análisis cuantitativo se expuso mediante la evolución de la velocidad, frecuencia de ciclo, longitud de ciclo e índice de ciclo. Por otro lado, se analizaron los diferentes factores que afectan a la correcta elección de la estrategia de paso, así como las más utilizadas en competiciones internacionales.

Palabras clave: técnica, piragüismo, estrategia de paso.
\end{abstract}

Abstract: The objective of this paper was to describe the new trends in technique analysis and pacing strategy in flatwater canoeing. Qualitative technique analysis were presented though out the descriptive and discriminative evaluation, while quantitative technique analysis were described by means of evolution of speed, cycle frequency, cycle length and cycle index. On the other hand, the different factors witch affects the right choice of pacing strategy and the most used race patterns in international competitions were analyzed.

Key words: technique, canoeing, pacing strategy.

\section{Introducción.}

El desarrollo de la técnica de paleo en piragüismo de aguas tranquilas ha sido realizado, tradicionalmente, mediante la observación y corrección del palista a lo largo de los entrenamientos. Por lo que se planteaba la necesidad de desarrollar formas más sistemáticas y reproducibles de valorar la técnica de paleo, ante esta situación Sánchez \& Magaz (1993), expusieron diferentes orientaciones para el análisis de la técnica, que posteriormente han sido desarrolladas y ampliadas en el aspecto cualitativo por las hojas de valoración de la técnica de paleo en kayak y canoa de Valcarce y Gómez (2005) y en las investigaciones sobre la evolución de las variables cinemáticas (Alacid, Ferrer, Martínez, \& Carrasco, 2005; Alacid et al., 2008) en el aspecto cuantitativo; en ambas ocasiones las valoraciones fueron orientadas hacia palistas de categoría infantil.

Por otro lado, la elección de la estrategia de paso óptima para cada distancia de competición y la amplia variedad de factores que influyen sobre ésta, supone uno de los principales problemas al diseñar la forma de competir en piragüismo de aguas tranquilas.

Por todo ello, este trabajo pretende describir las diferentes tendencias en el análisis de la técnica de paleo, así como en la determinación de la estrategia de paso más adecuada en piragüismo de aguas tranquilas.

\section{Análisis cualitativo de la técnica de paleo.}

Para Sánchez \& Magaz (1993), el análisis cualitativo de la técnica de paleo se puede realizar desde dos líneas diferentes de actuación:

- Valoración descriptiva: en la que se hacen valoraciones breves sobre el estilo del palista y su grado de aproximación al modelo técnico utilizado. Esta valoración aporta un gran número de detalles, pero requiere mucho tiempo para su realización y existen muchas dificultades a la hora de realizar comparaciones con otros palistas.

- Valoración discriminativa: en este tipo de valoraciones se aporta una puntuación numérica a cada una de las partes que se analiza en el gesto técnico, para conseguir al final del proceso una puntuación técnica global del palista. Este tipo de evaluación técnica es muy rápido de realizar, permite comparar a diferentes palistas o valorar la evolución de uno de ellos.

\footnotetext{
Fecha recepción: 02-09-08 - Fecha envío revisores: 03-09-08- Fecha de aceptación: 16-11-08 Correspondencia: Fernando Alacid Cárceles

No se puede afirmar que un tipo de valoración sea mejor que el otro, pues cada una de ellas persigue objetivos diferentes y pueden ser utilizadas de forma complementaria, incluso a éstas se puede añadir una valoración de tipo cuantitativo.

El tipo de observación utilizada, independientemente del método de evaluación cualitativa, puede ser la observación directa del palista en el desarrollo de la acción técnica de paleo o la grabación del paleo a ritmos elevados o de competición, para su posterior análisis.

Además de los aspectos técnicos relacionados con la disposición de los segmentos corporales, los indicadores cualitativos más importantes del piragüismo de aguas tranquilas, independientemente de que estemos hablando de kayak o canoa, son:

- La fluidez

- La continuidad y el ritmo

- La amplitud de palada (especialmente al principio de la misma)

Para identificar la fluidez, basta con observar la forma en que navega la embarcación, evitando movimientos de cabeceo y balanceo principalmente, que incrementen la resistencia al avance.

Para observar la continuidad y el ritmo en el paleo, identificaremos paradas y cambios de velocidad en el ciclo de paleo, especialmente entre la fase acuática y la aérea del mismo.

En la amplitud de palada en el ataque, buscaremos que el palista tenga la hoja de la pala sumergida totalmente en el punto más cercano a su ataque.

Mediante el uso de la valoración discriminativa, los autores (Sánchez \& Magaz, 1993) proponen 11 ítems de valoración técnica en kayak y 12 para canoa. Cada uno de ellos es valorado de 0 a 3 puntos en función de la aparición o ausencia de una parte determinada del gesto técnico o de las características de la navegación de la piragua.

Este tipo de análisis suele representarse en gráficos de tipo radial, donde la puntuación más alta se encuentra alejada del centro de la representación. De esta forma un palista con una técnica idéntica al modelo obtendrá la máxima puntuación en todos los ítems y su representación gráfica será la del polígono regular de número de lados equivalente al número de ítems valorados, utilizando las hojas de valoración técnica propuestas por Sánchez \& Magaz (1993), sería un endecágono para kayak y dodecágono para canoa.

Puesto que no hemos encontrado trabajos que desarrollen un análisis discriminativo de la técnica de paleo en aguas tranquilas, en la figura 1 exponemos un ejemplo de puntuación de dos canoistas tras realizar este tipo de valoración de su técnica de paleo. El palista representado por una línea continua posee una buena puntuación en aspectos relacionados con la fase acuática del paleo, teniendo que mejorar en el deslizamiento de la embarcación, en la continuidad y fluidez del gesto y en el ataque aéreo. Por otro lado, el palista representado por la línea discontinua 


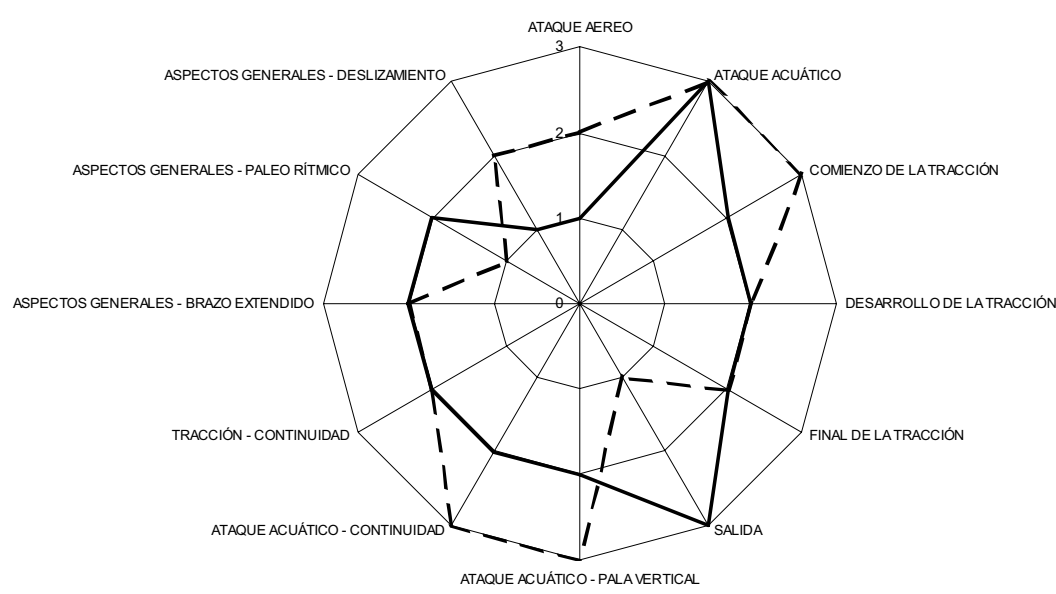

Figura 1. Ejemplo de valoración cualitativa de la técnica de paleo en canoa

uso de los datos obtenidos para aportar información a los palistas sobre su rendimiento, así como para planificar futuras carreras. Este método fue empleado por el equipo nacional alemán durante los Juegos Olímpicos de Barcelona en 1992, atribuyendo parte del éxito obtenido en la preparación del evento y durante la competición, al apoyo científico que se deriva de este tipo de análisis.

En palistas de categoría infantil, Cuesta, Polo \& Padilla (1991) estudiaron la correlación entre las marcas conseguidas sobre diferentes distancias de competición y diferentes parámetros fisiológicos obtenidos en kayakergómetro, aportando además algunos datos sobre la distancia recorrida por palada y la velocidad en el test de laboratorio.

Son las investigaciones realizadas en palistas de categoría infantil(Alacid et al., 2005; 2008) los primeros trabajos publicados en piragüismo sobre esta temática, aportando datos sobre la evolución de la velocidad, frecuencia, longitud e índice de ciclo en un test máximo

posee un buen comienzo de la palada, en lo que se refiere a las subfases de ataque y tracción, mientras que debería mejorar la salida de la pala y el ritmo de paleo, errores frecuentemente asociados en esta especialidad por tener que realizar el repaleo para mantener una dirección correcta.

\section{Análisis cuantitativo de la técnica de paleo.}

El análisis cuantitativo de la técnica en los deportes cíclicos es una herramienta que permite conocer la evolución de la velocidad a lo largo de la distancia de competición, así como de las variables que la condicionan (frecuencia y longitud de ciclo), aportando también datos para la búsqueda de la óptima relación entre las mismas, con el fin de conocer el efecto de la fatiga sobre el deportista y, por tanto, incrementar el rendimiento (Craig, Skehan, Pawelczyk, \& Boomer, 1985). La natación es el origen de este tipo de análisis en los deportes acuáticos, encontrando primer trabajo de investigación realizado por East (1970) a lo largo del campeonato neozelandés de natación. En un principio se investigó el comportamiento de la velocidad como producto de la frecuencia de ciclo y la longitud de ciclo con el fin de medir y evaluar la técnica de nado (Craig \& Pendergast, 1979; Keskinen, Tilli, \& Komi, 1989). Posteriormente, Costill, Kovaleski, Porter, Fielding, \& King (1985), introdujeron el concepto de índice de ciclo a partir del resultado del producto de la longitud de ciclo y la velocidad, como un índice de la eficiencia y economía de nado.

En el piragüismo de aguas tranquilas, las variables cinemáticas se suelen abordar por separado, analizándose el comportamiento de la velocidad sobre diferentes distancias de competición (Alacid \& Carrasco, 2004; Bishop, Bonetti, \& Dawson, 2002; Issurin, 1998), la comparación de la frecuencia de paleo en kayakergómetro y en agua (Alacid \& Carrasco, 2004; Barnes \&Adams, 1998; van Someren \& Oliver, 2002), así como en la navegación en la estela y en solitario (Gray, Matheson, \& McKenzie, 1995; Pérez-Landaluce, Rodríguez-Alonso, FernándezGarcía, Bustillo-Fernández, \& Terrados, 1998).

En la especialidad de slalom en aguas bravas también se ha analizado la cinemática de la técnica de paleo en un ciclo aislado (Caubet, 1999), para determinar las diferencias en el movimiento y en la posición de los segmentos corporales durante el paleo en agua y en un ergómetro específico, tanto en kayak como en canoa.

Sperlich \& Baker (2002) destacaron la importancia de obtener las variables cinemáticas durante los entrenamientos y competiciones. Para ello, propusieron la grabación del palista desde una motora o vehículo a lo largo de la distancia de competición, así como la utilización de las boyas del campo de regatas como referencia. Los autores proponen el de 500 y $200 \mathrm{~m}$ respectivamente, realizado por hombres y mujeres kayakistas (13-14 años) para la distancia de $500 \mathrm{~m}$, y añadiendo la categoría de hombres canoistas a las anteriores en el análisis de la distancia de $200 \mathrm{~m}$.

Para ello, se realizó una grabación lateral del palista a lo largo de la distancia de competición, ajustando en todo momento el encuadre de la cámara con la proa de la piragua al paso por las boyas, facilitando de esta forma la posterior obtención de los datos. Posteriormente se pasaron las grabaciones a formato de video digital, a 25 fotogramas por segundo.

El análisis cuantitativo se basa en la obtención de forma directa de la velocidad y la frecuencia de ciclo y mediante un sencillo cálculo la longitud de ciclo y el índice de ciclo. Teniendo en cuenta que la velocidad de una embarcación viene determinada por la distancia (metros) que recorre en un ciclo y la cantidad de ciclos que se realizan en una determinada unidad de tiempo (segundo), podemos determinar la siguiente ecuación:

- Velocidad $=$ Longitud de ciclo $($ metros/ciclo $) \times$ Frecuencia de ciclo (ciclos/segundo)

De esta ecuación, tras eliminar los ciclos, nos quedarían los metros por segundo: la velocidad. Para obtener la longitud de ciclo dividiremos la velocidad entre la frecuencia de ciclo.

Por otro lado el índice de ciclo se obtiene como el resultado del producto de la velocidad por la longitud de ciclo, esta variable ya fue definida por Costill et al. (1985) como un indicador de la eficiencia y economía en el ciclo en nadadores, pero nada indica que no pueda ser extrapolable al piragüismo. Por ejemplo, si tenemos a dos palistas que van a la misma velocidad pero uno de ellos avanza más metros en cada palada, esto indica que su palada es más efectiva y al multiplicar la velocidad por la longitud de ciclo sus valores del índice de ciclo serán superiores a los de su compañero.

En cuanto a la amplitud de los tramos a estudiar, se dividió la distancia en espacios lo suficientemente pequeños para obtener la mayor información sobre la evolución de la variable a lo largo de la prueba y lo suficientemente grandes para no analizar muchos tramos consecutivos en los que no existen variaciones de las variables cinemáticas. En trabajos previos (Alacid et al., 2005; 2008), se establecieron tramos de $100 \mathrm{~m}$ para una distancia total de $500 \mathrm{~m}$, exceptuando el primer parcial que se dividió en dos tramos de $50 \mathrm{~m}$ para conocer la influencia de la salida sobre los diferentes parámetros; mientras que para la distancia de $200 \mathrm{~m}$ se analizaron 4 tramos de $50 \mathrm{~m}$ cada uno.

Para obtener la velocidad, en primer lugar se determinaron los fotogramas en el que la proa de la embarcación se encontraba alineada con las dos boyas que marcaban el inicio y el fin del tramo a valorar. A 
continuación, se halló la diferencia entre los fotogramas transcurridos y tras dividirlo entre 25 , se obtuvo el tiempo empleado en recorrer el tramo. La velocidad resultó de dividir el espacio entre el tiempo, obteniendo los resultados en $\mathrm{m} \cdot \mathrm{s}^{-1}$.

Para calcular la frecuencia de ciclo, se contaron los ciclos completos realizados en el tramo, registrando los fotogramas en los que la hoja tomaba contacto con el agua, siendo aquéllos iguales o superiores a los seleccionados para determinar la velocidad, es decir, se tomó como referencia el primer ataque realizado tras completar el tramo. Tras obtener estos datos se dividió el número de ciclos entre el incremento de fotogramas dividido entre 25 , obteniendo los resultados expresados en ciclos $\cdot \mathrm{s}^{-1}$. Existen dos circunstancias especiales para la obtención de la frecuencia de ciclo: $1^{\mathrm{a}}$ ) en el primer tramo se desestimó el primer ciclo de paleo en el que se pone en marcha la embarcación, ya que éste se produce en condiciones en las que aquélla se encuentra parada, por lo que posee características diferentes al resto de los ciclos de paleo; $2^{\mathrm{a}}$ ) en el último tramo se utilizó el ataque previo a la entrada en meta, pues una vez concluida la prueba el palista suele dejar de palear.

La longitud de ciclo se obtuvo tras dividir la velocidad entre la frecuencia de ciclo obtenidas en un determinado tramo, expresando los resultados en $\mathrm{m} \cdot$ ciclo $^{-1}$. El índice de ciclo resultó del producto de la velocidad y la longitud de ciclo obtenidos en cada uno de los tramos, expresando sus resultados en metros ${ }^{2} \cdot(\text { segundo } \cdot \text { ciclos })^{-1}$.

Puede resultar novedosa para el entrenador de piragüismo la forma de expresar los datos obtenidos para las variables de frecuencia de ciclo y longitud de ciclo, ya que en vez de utilizar la palada como unidad, se utiliza el ciclo. Definiendo este último en piragüismo como dos paladas en kayak y una en canoa. Pero más nos puede sorprender el utilizar el segundo como medida temporal para expresar la frecuencia de ciclo, pero esto se realiza para simplificar posteriormente la obtención de la longitud de ciclo. De cualquier forma, pasar de ciclos por segundo a paladas por minuto responde a la sencilla operación de multiplicar por 120 .

\section{Estrategia de paso.}

Aunque la estrategia de paso y evolución de la velocidad responden a una misma realidad, podemos identificar el primero de los conceptos con la elección de la distribución óptima del esfuerzo del palista, en función de una serie de condicionantes tácticos como la distancia de competición, la climatología, etc. Mientras que la evolución de la velocidad hace referencia únicamente a las variaciones de esta magnitud a lo largo de una determinada distancia.

En el piragüismo de aguas tranquilas, dentro de sus modalidades olímpicas, se compite en distancias de 500 y 1000 m, en un campo de regatas en línea recta en el que se encuentran delimitadas 9 calles de $9 \mathrm{~m}$ cada una. La relación entre los palistas debe ser inexistente, y en el caso de que un palista se acerque a otro a lo largo de la competición, puede ser descalificado por intuirse que estaba navegando en la estela de otro competidor, suponiendo una ventaja desleal, tal y como establece el reglamento de aguas tranquilas de la Federación Internacional (ICF, 2007), y como ha sido demostrado por varios estudios (Gray et al., 1995; Pérez-Landaluce et al., 1998).

$\mathrm{Al}$ competir sin la influencia directa del resto de adversarios, los planteamientos estratégicos se centran principalmente en la evolución de la velocidad a lo largo de la distancia de competición, siendo éste el principal problema al cual los entrenadores se enfrentan frecuentemente, intentando obtener el mejor rendimiento mediante una optimización de los tiempos de paso (Alacid \& Carrasco, 2004).

Sánchez \& Magaz (1993) enumeran una serie de características de las competiciones en piragüismo que marcan la forma de competir, entre las cuales destacan, por un lado, la no existencia de condiciones estándar de competición y entrenamiento que permitan abordar el entrenamiento de ritmo de forma más rigurosa, relacionando intensidad y tiempo, o distancia y tiempo y, por otro lado, la salida o puesta en marcha de la piragua, que, hasta lograr la velocidad media de la prueba, supone un gasto energético suplementario que compromete el desarrollo posterior de la competición. Además, el estado del agua y la aparición de olas, producidas por las embarcaciones que van por delante, limitan la ventaja que se puede dar a los adversarios, puesto que dificulta el eficiente deslizamiento de la embarcación. A estas características hay que añadir el conocimiento de los oponentes, el análisis de pruebas anteriores propias y ajenas, el desarrollo de estrategias alternativas al planteamiento inicial, y la fijación del objetivo de cada competición (Cabases, 1991).

Para Issurin (1998) los factores que determinan la estrategia de paso en competición son los aspectos situacionales (la intervención del viento y las olas, el comportamiento del resto de competidores, etc.) y los factores programados (plan de rendimiento a lo largo de la prueba, como el aumento de ritmo en los últimos $200 \mathrm{~m}$, etc.).

Una vez se conocen las características más significativas de la competición, así como las de los deportistas, es preciso plantearse la elección de un modelo de estrategia de paso (Sánchez \& Magaz, 1993; Toro, 1986):

- Planteamiento regresivo: en el cual la velocidad va disminuyendo paulatinamente a lo largo de los parciales.

- Planteamiento uniforme: en el que la velocidad mantiene un comportamiento más estable, aunque el tramo más rápido es el más próximo a la salida.

- Planteamiento progresivo o negativo: en el que el tramo más rápido se encuentra al final del recorrido.

El planteamiento uniforme parece ser el más lógico con el fin de evitar el desgaste energético que suponen los cambios de velocidad a lo largo de la competición (Cabases, 1991; Hajossyr, 1987). Sin embargo, los palistas y los entrenadores prefieren una salida rápida, seguida de un planteamiento homogéneo a lo largo de la prueba (Bishop et al., 2002).

Para la distancia de $1000 \mathrm{~m}$, la necesaria uniformidad de la estrategia de paso se ve reforzada por la duración de la prueba (Cox, 1992). Para Sánchez y Magaz (1993), el primer tramo de $250 \mathrm{~m}$ es el intervalo más rápido de la distancia, mientras los otros tres mantienen una mayor uniformidad, produciéndose una disminución progresiva de la velocidad, siendo el tramo más lento el tercero o el cuarto. En este sentido, Alacid y Carrasco (2004) analizaron las finales de $1000 \mathrm{~m}$ del Campeonato del Mundo de 2003, encontrando un patrón decreciente de la estrategia de paso en todas las pruebas de embarcaciones individuales y dobles en las que se competía (K-1 y K-2 masculino, K-1 y K-2 femenino y C-1 y C-2 masculino), sin encontrar diferencias significativas entre estas modalidades.

Para Toro (1986) el planteamiento progresivo o negativo, practicado en otros deportes como la natación o el atletismo, es de muy rara aplicación en piragüismo debido a la influencia negativa de las olas generadas por las embarcaciones situadas en las primeras posiciones, produciendo problemas en la navegación y el equilibrio de la piragua. Este autor limita el uso de este tipo de estrategia de paso en pruebas de $1000 \mathrm{~m}$, tras realizar una buena salida, aplicando el planteamiento negativo en los últimos $500 \mathrm{~m}$.

Tabla 1. Estrategias utilizadas por los competidores y ganadores en Campeonatos del Mundo y Juegos Olimpicos. Tomado de Issurin (1998)

\begin{tabular}{|c|c|c|c|c|c|c|}
\hline \multirow{2}{*}{ Prueba } & \multirow{2}{*}{$\begin{array}{l}\text { Competidor / } \\
\text { Ganador }\end{array}$} & \multirow{2}{*}{$\mathrm{n}$} & \multicolumn{4}{|c|}{ Tipo de estrategia utilizada (\%) } \\
\hline & & & A & B & $\mathrm{C}$ & D \\
\hline \multirow{2}{*}{$\begin{array}{l}\text { Hombre K-1 } \\
500 \mathrm{~m}\end{array}$} & $\mathrm{C}$ & 69 & 40.6 & 36.2 & 13.0 & 10.1 \\
\hline & G & 12 & 50.0 & 41.7 & 8.3 & 0.0 \\
\hline \multirow{2}{*}{$\begin{array}{l}\text { Mujer K-1 } \\
500 \mathrm{~m}\end{array}$} & $\mathrm{C}$ & 76 & 24.0 & 31.0 & 12.0 & 9.0 \\
\hline & G & 13 & 46.1 & 38.5 & 15.4 & 0.0 \\
\hline \multirow{2}{*}{$\begin{array}{c}\text { Hombre K-1 } \\
1000 \mathrm{~m}\end{array}$} & $\mathrm{C}$ & 83 & 25.0 & 19.0 & 26.0 & 13.0 \\
\hline & G & 14 & 50.0 & 28.6 & 7.1 & 14.3 \\
\hline
\end{tabular}


Uno de los trabajos más completos sobre el planteamiento de las competiciones en piragüismo fue realizado por Issurin (1998), tras analizar 228 carreras en Campeonatos del Mundo y Juegos Olímpicos entre 1983 y 1997 encontró cuatro tipos de estrategias de paso asociadas a la evolución de la frecuencia de paleo (tabla 1):

A. Salida rápida, rendimiento uniforme en la mitad de la prueba e incremento de la velocidad y la frecuencia de ciclo al final de la prueba. Es la estrategia más popular entre los participantes, aproximadamente el 50\% de los ganadores usa este planteamiento y la mayoría de los competidores. El incremento de la velocidad y de la frecuencia de paleo al final de la prueba suele suceder en finales ajustados.

B. Velocidad y frecuencia de ciclo disminuyen progresivamente a lo largo de la distancia. También muy utilizada en competición. El descenso de las variables se produce porque el palista no puede incrementarlas al final de la distancia o no tiene necesidad de ello por la ventaja adquirida.

C. Salida rápida, rendimiento uniforme en la mitad de la prueba y descenso acusado de la frecuencia de ciclo al final de la prueba. Es una estrategia muy empleada en series clasificatorias cuando el palista tiene el paso asegurado a la final o se encuentra muy alejado de los puestos de clasificación y abandona.

D. Rendimiento uniforme a lo largo de toda la prueba. Es la menos popular de las estrategias, usada cuando el palista pretende ahorrar energía al inicio de la prueba y mantiene una velocidad y frecuencia de paleo uniforme en toda la distancia. Destaca la ausencia de ganadores usando este planteamiento en la distancia de $500 \mathrm{~m}$.

En resumen, el análisis de las estrategias de paso, aporta una información muy valiosa para los entrenadores y palistas, puesto que suponen una referencia hacia la que dirigir la planificación estratégica de las futuras competiciones.

\section{Bibliografía.}

Alacid, F., \& Carrasco, L. (2004). Distribución del esfuerzo en piragüismo sobre 1000 metros. Paper presented at the III Congreso de la Asociación Española de Ciencias del Deporte, Valencia.

Alacid, F., Ferrer, V., Martínez, E., \& Carrasco, L. (2005). Análisis cuantitativo de la técnica de paleo en kayakistas infantiles. Motricidad. European Journal of Human Movement(13), 133146.

Alacid, F., López-Miñarro, P. A., Ferragut, C., García, A., Ferrer, V., \& Martínez, I. (2008). Evolución y comparación de la velocidad, frecuencia, longitud e índice de ciclo sobre $200 \mathrm{~m}$, en palistas infantiles de diferentes modalidades. Motricidad. European Journal of Human Movement(20), 15-27.

Barnes, C. A., \& Adams, P. C. (1998). Reliability and criterion validity of a 120 s maximal sprint on a kayak ergometer.J Sports Sci, 16(1), 25-26.

Bishop, D., Bonetti, D., \& Dawson, B. (2002). The influence of pacing strategy on $\mathrm{VO}_{2}$ and supramaximal kayak performance. Med Sci Sports Exerc, 34(6), 1041-1047.

Cabases, J. M. (1991). Entrenamiento del ritmo. En Escuela Nacional de Entrenadores de Piragüismo (Ed.), Comunicaciones Técnicas VII (pp. 126-157). Madrid: Federación Española de Piragüismo.

Caubet, J.M.(1999). Estudio comparativo de los parámetros cinemáticos de la técnica de paleo, en un ergómetro de piragüismo y en agua. Apunts. Medicina de l'esport(131), 5-10.

Costill, D. L., Kovaleski, J., Porter, D., Fielding, R., \& King, D. (1985). Energy expendidure during front crawl swimming: prediction in middle distance events. Int J Sports Med, 6(5), 266-270.

Cox, R. W. (1992). The science of canoeing. Cheshire: Coxburn Press.

Craig, A. B., Jr., \& Pendergast, D. R. (1979). Relationships of stroke rate, distance per stroke, and velocity in competitive swimming. Med Sci Sports, 11(3), 278-283.
Craig, A. B., Jr., Skehan, P. L., Pawelczyk, J. A., \& Boomer, W. L. (1985). Velocity, stroke rate, and distance per stroke during elite swimming competition. Med Sci Sports Exerc, 17(6), 625-634.

Cuesta, G, Polo, J. M., \& Padilla, S. (1991). Correlación entre la marca deportiva obtenida en test de campo y parámetros fisiológicos obtenidos en laboratorio, en piragüistas adolescentes. Apunts, 18, 130-142.

East, D. J. (1970). An analysis of stroke frequency, stroke length and performance. New ZealandJ Health Phys EdRecr(3), 16-27.

Gray, G. L., Matheson, G. O., \& McKenzie, D. C. (1995). The metabolic cost of two kayaking techniques. Int J Sports Med, 16(4), 250-254.

Hajossyr, J. (1987). Modelisation de la tactique en competition de canoe - kayak. / A model for strategy during canoe-kayak competition. Traductions INSEP(612), 1-11.

ICF. (2007). Flatwater racing competition rules. Consultado el 14-062007, disponible en www.canoeicf.com

Issurin, V. (1998). Analysis of the race strategy of world-class kayakers. En V. Issurin(Ed.), Science \& practice of canoe/kayak high-performance training: selected articles in memory of junior world champion Nevo Eitan (pp. 27-39). Tel-Aviv: Elite Sport Department of Israel.

Keskinen, K., Tilli, L. J., \& Komi, P. (1989). Maximum velocity swimming: interrelationships of stroking characteristics, force production and anthropometric variables. Scan J Sport Sci, 11(2), 87-92.

Pérez-Landaluce, J., Rodríguez-Alonso, M., Fernández-García, B., Bustillo-Fernández, E., \& Terrados, N. (1998). Importance of wash riding in kayaking training and competition. Med Sci Sports Exerc, 30(12), 1721-1724.

Sánchez, J. L., \& Magaz, S. (1993). La Técnica. En J.L. Sánchez(Ed.), Piragüismo (I) (pp. 101-386). Madrid: COE.

Sperlich,J., \& Baker,J.D. (2002). Biomechanical testing in elite canoeing. En K. E. Gianikellis (Ed.), Scientific Proceedings of the XXth International Symposium on Biomechanics in Sports (pp. 44-47). Cáceres: Universidad de Extremadura.

Toro,A.(1986). Canoeing: an olympic sport. San Francisco: Olympian Graphics.

Valcarce, F., \& Gómez, J. (2005). Fichas de corrección de la técnica de paleo en kayak y en canoa: Federación Madrileña de Piragüismo.

Van Someren, K. A., \& Oliver, J. E. (2002). The efficacy of ergometry determined heart rates for flatwater kayak training. Int $J$ Sports Med, 23(1), 28-32. 